\title{
Forty hours of declarative programming Teaching Prolog at the Junior College Utrecht
}

\author{
Jurriën Stutterheim Wouter Swierstra Doaitse Swierstra \\ Department of Information and Computing Sciences \\ Universiteit Utrecht \\ P.O.Box 80.089, 3508 TB Utrecht, The Netherlands \\ $\{$ j.stutterheim,w.s.swierstra, doaitse\}@uu.nl
}

\begin{abstract}
This paper documents our experience using declarative languages to give secondary school students a first taste of Computer Science. The course aims to teach students a bit about programming in Prolog, but also exposes them to important Computer Science concepts, such as unification or searching strategies. Using Haskell's Snap Framework in combination with our own NanoProlog library, we have developed a web application to teach this course.
\end{abstract}

\section{Introduction}

The Junior College Utrecht (JCU) is a joint initiative of Utrecht University and 26 local secondary schools. The JCU offers talented students already interested in science a choice of several short courses at several different departments of the Faculty of Science of Utrecht University. In their penultimate year of secondary school—when the students are approximately seventeen years old—students may do a small research project that encompasses approximately 40 hours of work, including the preparation of a final report and a presentation. Students spend two and a half days performing experiments, followed by two days of preparing their final presentation and report. On the last afternoon, students present their projects to one another. The courses consist of five full days, spread over six weeks. The students may indicate their preference for several different topics, including biology, veterinary science, chemistry, or mathematics. Finally, the students are distributed over the available courses, taking their preferences and the available space on each course into account. In the past two years, we have developed a Computer Science course for these students [20].

Choosing suitable material for such a course is not easy. Part of the purpose of the JCU is to ignite the students' interest in (Computer) Science. While we want the students to learn a bit of computer programming, the course should strive to teach more. We would like our students to learn something about algorithms, semantics, programming language design, and other abstract concepts from Computer Science. In our experience, very few students who start the course have a clear idea of what Computer Science is. One of our main goals was to ensure that students that complete our course learn what Computer Science is and how it differs from Mathematics and other Sciences. Finally, we hope to encourage students to enrol at Utrecht University - above all else, the course should be fun.

Early on, we decided that we wanted to teach the JCU students to program. Seeing your own code execute on your computer for the first time is a magical experience. Suddenly students become aware that they can instruct the computer to do what they tell it. Instead of simply consuming the applications others have written, students learn that a computer is a programmable device that they can control themselves.

Once we decided that we wanted to teach a programming language, the next question is of course which language to teach. Should we teach a mainstream programming language, such as Java or C\#? Or should we teach a 'toy' language such as Alice [2] or Scratch [14]? Or should we teach languages

M. Morazán and P. Achten (Eds.): Trends in Functional

Programming in Education 2012 (TFPIE 2012).

EPTCS 106, 2013, pp. 50-62 doi 10.4204/EPTCS.106.4 
popular in the functional programming language research community such as Agda [16], Epigram [15], OCaml [11], Racket [9], or Haskell [17]? We opted for none of the above.

The following requirements helped us to determine our choice of language:

- The language needs to be simple. Students should be up and running as quickly as possible. As we only have approximately two and a half days to teach the students, we want a language with minimal opportunity for making syntax errors or type errors, and getting incomprehensible compiler messages as a result.

- The language needs to be non-trivial. Many introductory programming languages target a younger, pre-teen audience by offering drag \& drop IDEs and focussing on drawing and/or animating images. The language should be challenging enough to intrigue clever, seventeen year-old secondary school students. A language like Scratch, for example, is great for simple animations and games, but it does not illustrate what real Computer Science is about and how real-world problems can be solved by programming.

- The language needs to create a level playing field. Some students already have programming experience when starting the JCU course. This can be extremely discouraging for students without prior programming experience. Inexperienced students may get the (incorrect) impression that they are not suitable for a degree in Computer Science.

- The students have no experience with Computer Science. The Mathematics course taught at their secondary schools, where they learn some elementary calculus and geometry, is the closest related subject. To engage the students, the language we choose should relate to what they already know.

In the end, we settled upon (a fragment of) Prolog. This may seem like a peculiar choice, so we would like to provide some further motivation for this decision:

- The students have no trouble learning the syntax. Typically, they are writing their first Prolog relations within hours. Had we tried to teach Java, for example, we would need a significant amount of time to explain what public static void means and how to deal with curly braces and semi-colons. Choosing Prolog avoids these problems altogether.

- They can solve non-trivial problems by writing out a precise, mathematical specification. In our course, for instance, the students develop a small Sudoku solver. After the students have developed a specification of what a valid solution to a Sudoku problem is, Prologs search automatically provides them with a Sudoku solver. In the remainder of the course, the students can cover a wide variety of problem domains, including scheduling problems, routing problems, basic genetics, and logical brainteasers. In each of these domains, they learn how to formalize some abstract concept, resulting in an executable program.

- Using Prolog gives us the opportunity to teach the students about backtracking, unification, proof trees, and many other important concepts. We hope that by doing so, they learn there is more to Computer Science than 'just programming.'

- We can teach them how an interpreter works. We have implemented a minimal interpreter for Prolog in Haskell, and use this to teach our students a bit about functional programming. Not all students study this material, but the students with previous programming experience find this by far the most interesting part of the course.

- Prolog has no type system. While reasoning about types is an important aspect in the functional programming courses at Utrecht University, teaching the JCU students about types and have them 
write interesting programs within 40 hours seems unrealistic. In Prolog, the students can easily write an run their programs without having to decipher type-error messages first.

We have developed a web application to support this course. The application consists of two modes of operation: a web-based interface to our Prolog interpreter and an interactive Prolog proof assistant. This application, including its browser-based front-end, was built entirely in Haskell using the Snap web framework and the Utrecht Haskell Compiler (UHC). In the following sections we will discuss the structure of our course (Section 2), the implementation of our Prolog interpreter (Section 3), the interactive proof assistant (Section 4), and the students' experience working on this course, using these tools (Section 5).

\section{Course setup}

\subsection{Planning}

As the title of this paper suggests, we only have a very limited amount of time to teach this course. Unfortunately, this time constraint is put in place by the JCU itself, and is not something we as teachers of an individual pre-thesis track can easily change. In practice, we only have five days of about 6 to 7.5 hours each to introduce the students to Prolog, and assist them in doing their research project, preparing their presentation, and writing their report. The entire course is roughly structured as follows:

Day 1 We meet the students for the first time. After a short introduction, the students form teams of preferably two, but if need be three people. With the teams formed, they start reading our lecture notes [20] and start working on the exercises we have prepared.

The exercises start off slowly with an introduction to substitutions. Afterwards, the lecture notes continue with an introduction to Prolog and depth-first search. The lecture notes conclude with an explanation of how our Haskell implementation of a Prolog interpreter works. Before students can study this chapter, they are referred to the first two chapters of Learn You a Haskell [12]. In practice, very few of students get around to the Haskell part of the course.

Day 2 The students get some time to finish working on the lecture notes and ask questions about it. Afterwards, each team selects a research project and starts working on it. Students can come up with their own project, but since they generally cannot correctly determine the scope of a project themselves, we present them with a list of projects from which to choose.

Day 3 Work continues on the research project and the students start working on a report in which they detail what they have learnt during the course. This report is mostly aimed at the course's teachers and is expected to discuss the technical implementation details of their research project. In addition, the students are asked to reflect on how they executed their research and how they might improve in a future project.

Day 4 In addition to continuing work on their report, the students start working on their presentation. The presentation needs to be aimed at a general audience of parents, teachers from other disciplines, and fellow students that have chosen other topics at the JCU. In practice, the students utilize this day to continue work on their research project as well. 
Day 5 At the end of the day, the students will need to present their projects to a general audience of both parents and teachers, hence this day is mostly concerned with practising and improving the presentations.

One week after the final presentations, the students have to hand in their report.

\subsection{Course material}

The lecture notes provided to the students provides them with an introduction to Prolog and its implementation. The course notes start with a short introduction on substitutions, equations, and variables in terms of the high-school mathematics with which the students are already familiar. On the surface, this has little to do with programming, but it aims to fix some terminology before introducing Prolog.

Next, they learn a bit about Prolog through a series of examples, starting with a set of relations to describe a family tree. The first queries they write themselves are along the lines of "Who are the children of Alice?' or 'Who are Bob's grandparents?'. By practicing with these simple examples, they gain some basic familiarity with Prolog, but also learn the difference between existential and universal quantification. To understand simple rules such as:

grandparent $(X, Y):-$ parent $(X, Z)$, parent $(Z, Y)$.

You already need to understand that $X$ and $Y$ are universally quantified and that $Z$ is existentially quantified.

We introduce recursion very early on, starting with a definition of an ancestors relation. We then move on to simple Peano arithmetic, using recursion to define addition and multiplication.

Once they have seen Peano arithmetic, the next step is to study simple list processing. We show how to define simple functions such as length and append in Prolog. This all builds up to a (mini) Sudoku solver. This Sudoku solver itself merely formally describes what a solution is, leaving the search to Prolog's resolution mechanism. We start by defining predicates that state that a given list contains no duplicates. Using this, we define that all the rows and columns of a given board have no duplicates. It is then up to the students to complete the solver, by defining what it means for the 'boxes' of a Sudoku puzzle to have no duplicates.

Having completed the Sudoku solver, the course notes shift towards describing how Prolog resolution works. At first, we present a pseudo-code algorithm, exposing the students to concepts such as unification, search trees, and depth-first search. The final chapter of the notes explains how this pseudo-code can be implemented in Haskell.

\subsection{Projects}

Once the students have worked their way through the lecture notes, they start their own short research project. The research projects take the form of a more open ended challenge. Many students choose to use the Prolog interpreter already present on the lab machines, rather than use the web interface. This allows them to use a regular text editor to write their programs and use some of the features of Prolog that our simple interpreter does not support, such as special syntax for natural numbers.

The projects they work on are quite varied. Some are inspired by Sudoku-like puzzles, such as Kakuro. Others build on the family tree example, asking them to define new family relations or to extend the relations with some basic genetics (e.g., if both your parents exhibit the same recessive trait, you share that trait). Furthermore, we offer them a choice of several other Prolog programming task, such as defining a toy route planner system or simple scheduling software. Finally, we suggest several projects 
that modify the interpreter, such as implementing a breadth-first search or adding a cut operator. These last exercises tend to be quite daunting.

To give you a better idea of the kind of project the students write, we will briefly discuss the routing software that one team wrote last year. They started with having to define a binary relation between a fixed number of cities that represents that two cities are immediately connected. They then extended this to a recursive relation that holds if two cities are connected by zero or more steps. This is a bit subtle: you need to keep track of a list of cities that you have already visited to ensure that you do not loop. Finally, we asked the students to extend this simple algorithm to also compute the shortest path using iterative deepening.

All in all, the students tend to write a small amount of code; a project is typically between 30 to 100 lines of Prolog. In the bigger programs, a large part of the code often consists of enumerations of various base cases. The students find it challenging to have to start from scratch, but at the same time they enjoy the creative freedom associated with programming.

\section{NanoProlog Interpreter}

To allow students to play with Prolog, and-for the really fast learners-to learn how a Prolog interpreter works, we have written a small Prolog interpreter of our own, called NanoProlog. The core of our interpreter consists of 150 lines of Haskell, excluding the parser and main I/O loop. This section aims to give you an idea of how our NanoProlog interpreter works. The complete source code is available from Hackage as the NanoProlog package. We hope that it is simple enough that motivated students, with our help, will be able to understand how this interpreter implements Prolog's backtracking search.

The central data types we use to represent Prolog programs are defined as follows:

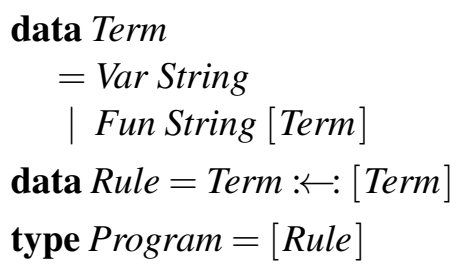

A Prolog Term is either a variable or a function constant applied to a list of terms. To simplify parsing, we require all variables to start with a capital letter; all constants should start with a lower-case letter. A Rule describes a single Prolog inference rule. The rule $t: \leftarrow$ : $s$ states that to prove the goal $t$, it is sufficient to prove each sub-goal in the list $t s$. For example, a Prolog rule such as

ancestor $(X, Y)$ :-parent $(Z, Y)$, ancestor $(X, Z)$

will be parsed as:

$$
\begin{aligned}
& \text { (Fun "ancestor" [ Var "X", Var "Y"]) : } \\
& \text { [Fun "parent" [Var "Z", Var "Y"] } \\
& \text {,Fun "ancestor" [Var "Z", Var "Y"]] }
\end{aligned}
$$

Finally, a program consists of a list of such rules.

The Prolog interpreter takes a program as input, together with a goal query. It constructs an inhabitant of the following Result type: 
newtype Env $=$ Env $\{$ fromEnv :: Map String Term $\}$

\author{
data Result \\ = Done Env \\ | Apply $[$ Result $]$
}

The answers we are looking for are substitutions, mapping variables to Prolog terms. We represent such substitutions as environments, using Haskell's Data.Map library. If the answer to our query is simple enough, there might not be any variables and the environment may be empty. The Result data type represents the search tree that is built up during resolution. It has two constructors: the Done constructor returns the required solution; the Apply branches over all possible rules that can be applied to solve the current goal.

The resolution process now proceeds in two steps: we begin by constructing a Result data type. By traversing this tree in any order, we can search for answers.

The solve function below forms the heart of our interpreter. The function unifies the current goal with the conclusion of every possible rule. When unification succeeds, we proceed by resolving the right-hand side of the rule, together with any remaining goals. When an Apply node does not have any children, this represents a 'dead-end' with no possible solutions.

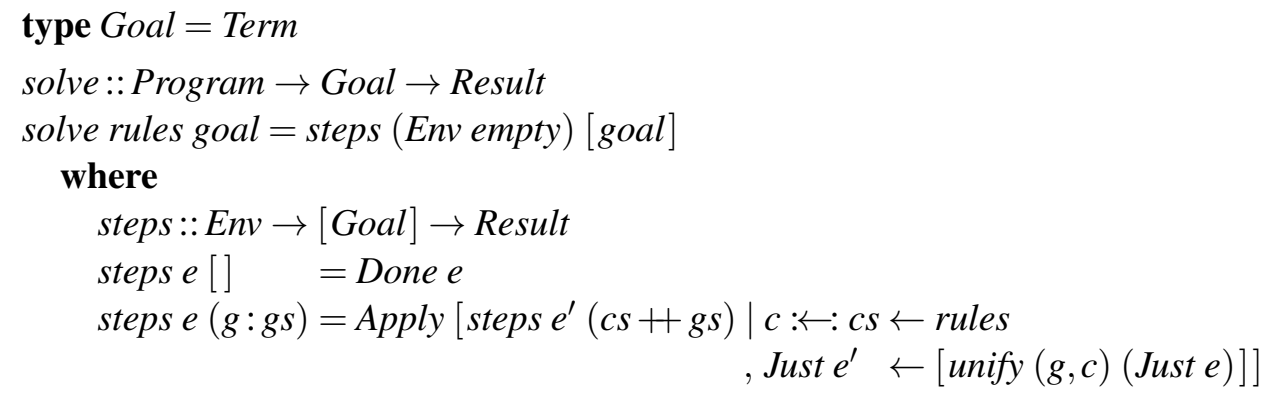

The actual implementation also tracks which rules are applied at every step, so that we can not only return the successful substitutions, but also the trace of all the rules that were applied to construct this solution.

The unify code is shown below. When the unify function is applied to two terms and a non-empty environment, the two terms are unified after the variables in the terms have been substituted by values from the environment. If one of the two terms is a variable, a substitution from the variable to the other term is inserted in the environment. If both terms are a Fun, the right-hand sides of the rules are unified if and only if both rules have the same name and the same number of terms on the right-hand side.

$$
\begin{aligned}
& \text { unify :: }(\text { Term, Term }) \rightarrow \text { Maybe Env } \rightarrow \text { Maybe Env } \\
& \text { unify_ Nothing = Nothing } \\
& \text { unify }(t, u) \text { env@(Juste@(Env m) })=\text { uni (subste t) (subste u) } \\
& \text { where } \\
& \text { uni }(\operatorname{Var} x) \quad y \quad=\text { Just }(\text { Env }(\text { insert } x \text { y } m)) \\
& \text { uni } x \quad \text { (Var } y) \quad=\text { Just }(\text { Env }(\text { insert } y x \text { m })) \\
& \text { uni (Fun xxs) (Fun y ys) } \\
& \mid x \equiv y \wedge \text { length } x s \equiv \text { length } y s=\text { foldr unify env }(z i p x s \text { ys }) \\
& \text { uni } \text { u }_{-} \quad \text { = Nothing }
\end{aligned}
$$


The Subst typeclass is used to overload the subst function to work on terms, lists of terms, and rules.

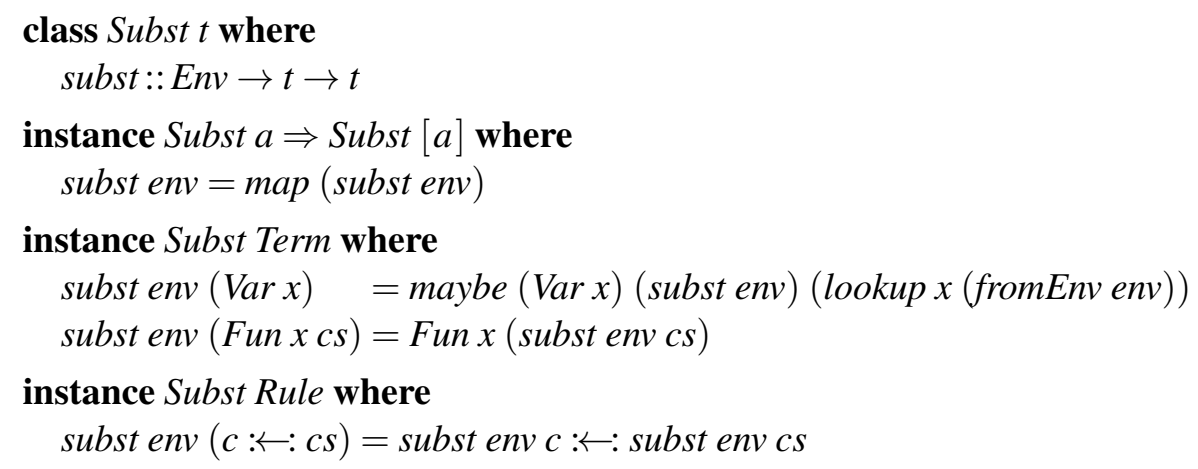

Our NanoProlog interpreter does have several restrictions. There is no cut operator; there is no way to define negation; there are no built-in integers, strings, lists, IO, or other functionality. To keep the implementation minimal, there is no occurs check. Yet the resulting, simple language is just enough to solve simple, but interesting problems.

\section{Web Application}

To allow the students to experiment with Prolog, without having to install any software themselves, we have developed a web application. The application has two modes of operation: as an interactive 'theorem prover' and as a Prolog interpreter.

In the first mode, we aim to teach the students how unification and backtracking work. Students can enter a Prolog query and then try to 'prove' it themselves by dragging and dropping rules from a list onto the query, thereby constructing a proof tree. Dropping a rule on a term unifies the conclusion of the rule with that term. The body of the rule may then introduce new sub-goals, expanding the proof tree. This is repeated until all the proof tree's leaves are basic Prolog facts, and the proof is complete.

A student can ask for feedback while working on the proof. When a branch of the tree still has open sub-goals, its leaves are rendered with a yellow background, indicating that there is still some work to be done. Nodes that have been successfully completed turn green. When all nodes have turned green, the proof is complete and the student is congratulated with a message box. Figure 1 on the facing page shows an incomplete proof. The root node has successfully been proved, but its children still contain open goals. By dragging and dropping the rules, listed on the right, on the open sub-goals the students can complete the proof.

Variables can be substituted in two ways. When a rule's conclusion is unified with an open sub goal, a substitution is produced and applied to the entire tree. Alternatively, the students can manually apply a substitution by using the controls just below the proof tree.

The second way the students can use the application is by using it as an interpreter. Figure 2 on page 58 illustrates this mode of development. Students are presented with a single text field in which they can enter a Prolog query. If the query can be proven, using the rules on the right, the interpreter will print the corresponding proof trees and substitutions on the screen. Students can define new rules, using the text field at the bottom-right.

Before being able to use the application, students need to register. This enables us to store a set of rules for each individual student, giving them their own playground to experiment with Prolog. Having a web application that the students can access from home is a huge advantage. The students do not need 


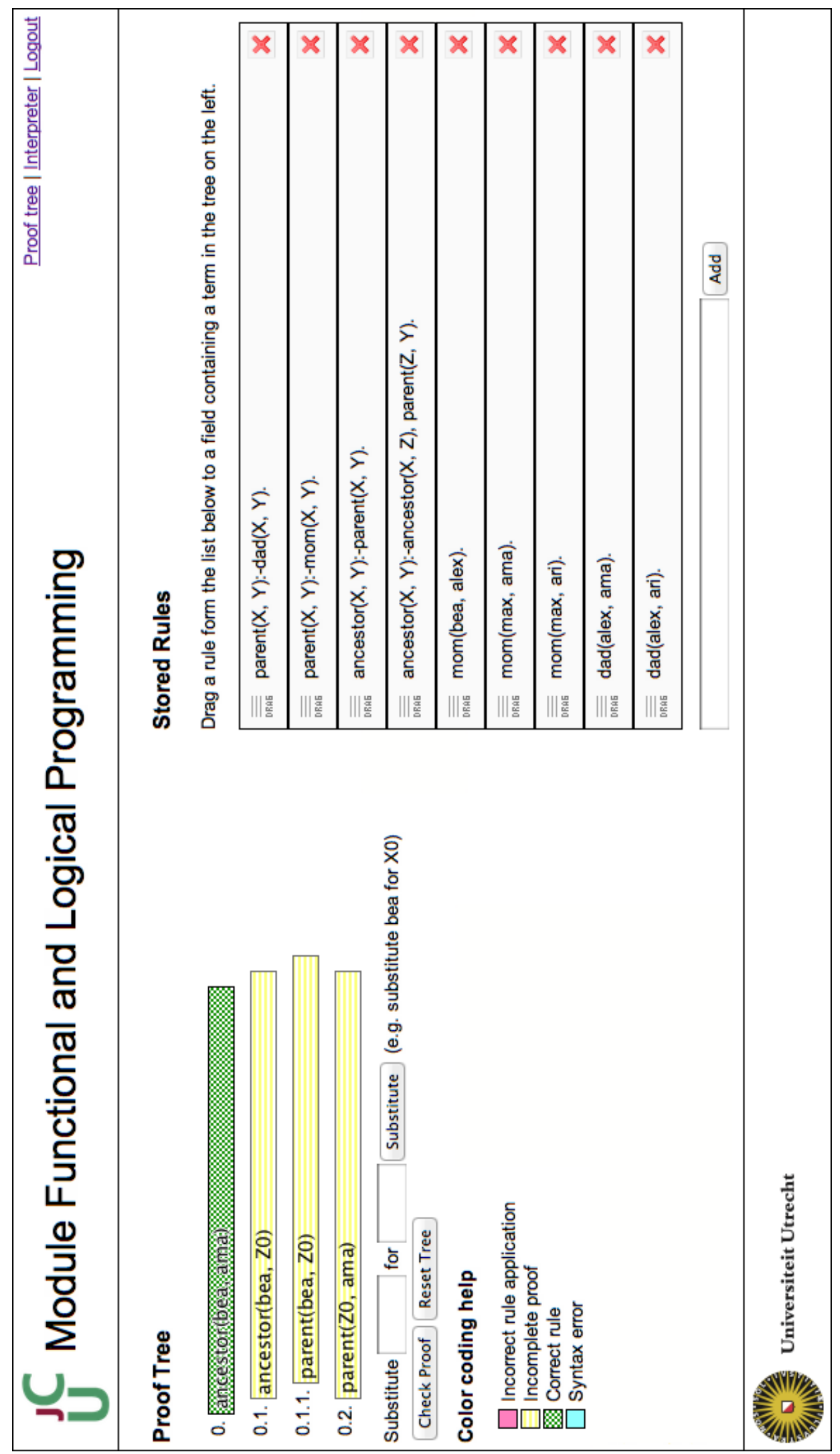

Figure 1: An incomplete proof 


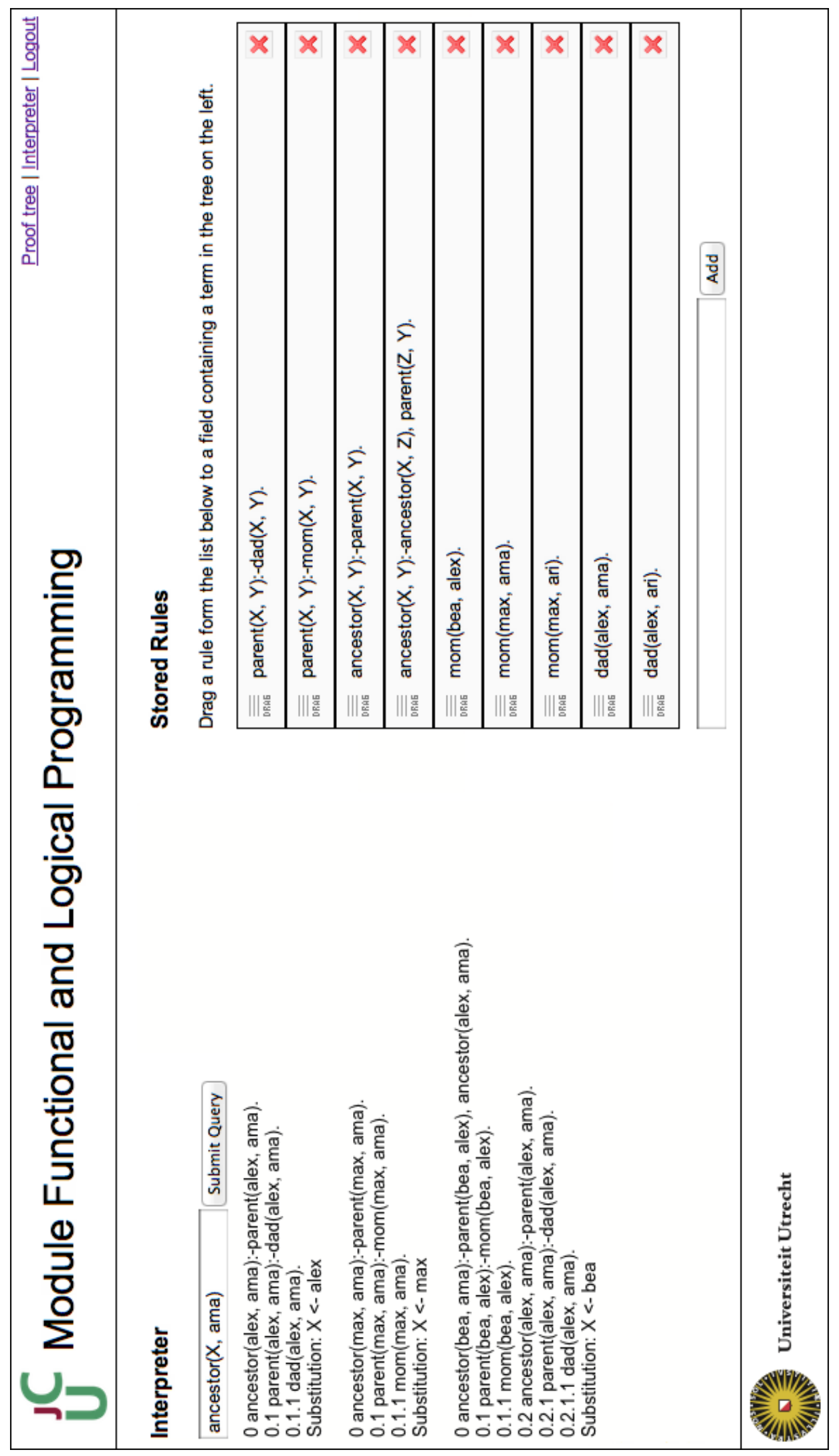

Figure 2: A proof found by the web-based interpreter 
to install their software and can experiment freely with our tools. For many of the larger projects the students do use SWI Prolog, that is already installed on the university computers.

\section{Implementation}

We have implemented both the client and the server part of the application entirely in Haskell. On the server side, we use the Snap Framework [1] to expose a RESTful [8] API to the NanoProlog interpreter and a database in which we store the Prolog rules the user has defined.

On the client side, we make use of the Utrecht Haskell Compiler's (UHC) [5, 4] JavaScript backend [6]. This enables us to write Haskell code and cross-compile it to JavaScript, which is run in the students' browser. Earlier revisions of the software were implemented in plain JavaScript and delegated parsing terms, unification and proof verification to the NanoProlog library on the server. By compiling the NanoProlog library to JavaScript, these operations can be executed client-side, eliminating additional AJAX requests. The 'theorem prover' part of the application - the first mode of operation-is implemented completely in Haskell and is running entirely on the client. Unfortunately, the JavaScript code generated by the UHC is not fast enough to run the graphical user interface to the interpreter - the second mode of operation - on the client completely. Instead, the user interface delegates the queries entered by the user to the server, where the much faster native code interprets them and sends the results back to the client.

\section{Discussion}

\section{Results}

As we mentioned in Section 2, we only have about two and a half days to teach the students. The remaining time is reserved for the preparation of a presentation, the writing of a final report, and a mini-symposium where all students across the different sciences present their results.

The first year we ran this course, it was quite 'free-form', in part because we had not assembled all the required material. Instead of spoon-feeding the students, we hoped that they would be capable of some individual exploration: the JCU specifically aims to teach students how scientific research works. In practice, however, these students, regardless of how motivated they are, are too young to work independently.

The second time we ran the course, we provided the students much more structure. We had prepared a set of course notes with numerous small exercises. This gave the students a clear initial goal: read the course notes, complete the exercises, and learn to use the web application. After completing the notes, the students embarked on their research projects. This gave the students the freedom to work on a small research project, but still provides enough guidance.

Student Reflection Throughout the course, the students were under constant supervision, which allowed them to frequently ask questions. While working through the course notes, the concept that the students seem to struggle with most was the scope of variables. The students found it hard to keep in mind that a variable $X$ in one rule is not the same variable $X$ in another rule, especially in the case of recursion. Luckily, by the time they moved on to their projects, this confusion had nearly completely disappeared. 
At the end of their research project, all of the students are required to reflect on their research by writing a short report. We summarize the experiences of the spring 2011 class here, which consisted of eleven students. Some of them had some prior programming experience, but most did not.

Especially the students without programming experience indicated that they did not really know what Computer Science was or how difficult the course was going to be. Some even indicated that this was an important reason for choosing to take part in this project. Despite the lack of prior experience, nearly all of the students enjoyed the course, even if they found it to be quite challenging. Many students would have liked to have had more time. They would have liked to get a better understanding of what they were doing and to be able to do more programming.

Even though the web application and interpreter were available for exploring Prolog, the students indicated that they were glad that they had the opportunity to ask questions in class.

The spring 2012 class had more extensive lecture notes to work with, which also contained several exercises. The students whom we spoke to indicated that this greatly helped them in understanding the theory. Half of those students also indicated that taking part in this course made them strongly consider choosing a Computer Science education.

Our experience Having run the course for two years now, we would like to reflect further on our experience. First of all, is Prolog a good choice? In the introduction we motivated our choice for Prolog: it is a simple language with interesting semantics, that we expect very few students to have studied already. In our experience, students enjoyed learning Prolog. They get started almost immediately as the syntax is extremely lightweight. Students can get frustrated at times-many are not used to the precision necessary to write computer programs. Missing parentheses are an all too common mistake. Perhaps better IDE support or a structure editor would help.

Students enjoy learning to program. They enjoy the intellectual challenge and seeing their code work. Unfortunately, once they have finished their projects, we think it is unlikely they will continue programming in Prolog. One student explicitly asked: what language do I learn next? This is a clear drawback of our choice for Prolog. Teaching a language such as JavaScript, that they have running in their browser already, would increase the chance that the students continue programming at home. The downside of using JavaScript, however, is the higher barrier to entry. We would need to spend more time explaining syntax, for instance. Perhaps the Racket approach would work well here: starting with a simple language (fragment) that is incrementally extended to a general purpose programming language.

One reviewer asked if it was too easy to guess the solution to the Prolog exercises. This was not our experience at all! In the interactive proof assistant sessions, where students need to assemble a derivation, this may be the case. The students can try various rules until they find one that fits. Even if they do produce derivations by trial and error, we find that seeing the unification happening before their eyes can still be instructive. The Prolog predicates we ask them to write are much harder to get right by trial and error. As soon as any recursion is involved, we believe the chances of guessing the right solution are very low.

We aimed to teach the students programming and Computer Science. How much do they really learn in the limited time we have available? After completing the course, the students have written a small program that computes something interesting starting from scratch. In a sense, they have learned to program. Of course, they have plenty to learn before they can write any kind of application, but we feel that all the students take their first steps in computer programming, however small these may be. Did the students learn about Computer Science? Yes and no. As we mentioned above, all the students finish the course with a much clearer idea of what Computer Science is. They are exposed to concepts such as 
algorithms and search trees, but they would need further practice to understand these abstract concepts completely. For example, we believe that most students could visit a tree in depth-first order, but very few could give an accurate pseudo code implementation of depth-first search.

Finally, we learned the importance of setting up a stable teaching environment. Despite having tested our web application before the project started, students still managed to break things in unexpected ways. Having SWI Prolog installed on the machines was a good fall-back option.

\section{Related Work}

There are several similar initiatives aimed at teaching programming to secondary school students. Bootstrap [18] is a curriculum aimed at middle-school children of ages 11 to 14. It focuses on teaching kids how to program video games using algebraic and geometric concepts that they know from their mathematics classes. As such, the curriculum is designed to work in conjunction with the regular middle-school program. It consists of nine units and some supplemental lessons. Students work with the functional programming languages Racket and Scheme. It also offers a web-based environment in which the children can write and execute code and see the result appear in the web browser.

Haskell for Kids [19] targets a similar audience as Bootstrap, namely children around the age of 12 or 13. It teaches the basics of Haskell programming by letting the children draw images and even animations using the Gloss library [13]. Similarly to bootstrap, Haskell for Kids offers a web-based development interface.

"How to Design Programs" [7] is a book with content similar to our curriculum. It teaches Scheme as a core subject for a liberal arts education. It differs not only in the target audience, but also in focus: it teaches the student to write programs, with underlying concepts as an aside, whereas we focus on teaching the student about the underlying concepts with learning how to program as an aside.

Khan Academy [10] offers a collection of introductory video lectures on Python. Exercises are not included. The target audience is not specified, but the level of the videos suggests that it is aimed at teenagers and above.

Alice [2] takes a more graphical approach to teaching programming. It focusses on visual learners by enabling them to drag and drop objects into a program to create an animation or a game. As opposed to the previous works, it uses a Java-like language, wrapped in a graphical user interface of a custom IDE. It is targeted at post-secondary education students.

Scratch [14] provides a drag and drop interface to programming in a custom IDE. The language itself is imperative, with an event-driven concurrency model. The language has a simple, but intuitive type system that shows types as different shapes. Scratch is aimed at children between 8 and 16 years of age.

Microsoft have developed Small Basic [3] to encourage people to learn how to program. Small Basic is a simplified Basic dialect. The IDE is simple and intuitive, and focuses on providing information about the Small Basic libraries API. It has a similar target audience to Khan Academy.

\section{Conclusion}

The module we teach is aimed at secondary school students. Besides teaching them a bit about computer programming, we aim to teach them about Computer Science. We expose them to concepts such as unification and substitution; explain search trees and search algorithms; and teach them to solve problems using recursion. We then proceed to show them how these concepts are implemented in an interpreter by showing an implementation in Haskell. We feel that this really distinguishes our course from many of the existing approaches already available. 


\section{References}

[1] Gregory Collins, Doug Beardsley, Shu-yu Guo, James Sanders, Carl Howells, Shane O'Brien, Ozgun Ataman, Chris Smith \& Jurriën Stutterheim: Snap Framework. Available at http://snapframework.com.

[2] Matthew J. Conway (1997): Alice: Easy-to-Learn 3D Scripting for Novices. Ph.D. thesis, University of Virginia.

[3] Microsoft Corporation: Microsoft Small Basic: An introduction to programming. Tutorial available online.

[4] Atze Dijkstra, Jeroen Fokker \& S. Doaitse Swierstra (2009): The Architecture of the Utrecht Haskell Compiler. In: Haskell Symposium, doi $10.1145 / 1596638.1596650$.

[5] Atze Dijkstra, Jeroen Fokker \& S. Doaitse Swierstra (2009): UHC Utrecht Haskell Compiler. Available at http://www.cs.uu.nl/wiki/UHC.

[6] Atze Dijkstra, Jurriën Stutterheim, Alessandro Vermeulen \& S. Doaitse Swierstra: Building JavaScript applications with Haskell. Accepted for IFL 2012.

[7] Matthias Felleisen, Robert Bruce Findler, Matthew Flatt \& Shriram Krishnamurthi (2003): How to Design Programs - An Introduction to Programming and Computing.

[8] Roy Thomas Fielding (2000): Architectural Styles and the Design of Network-based Software Architectures. Ph.D. thesis, University of California, Irvine.

[9] M. Flatt (2010): PLT. Reference: Racket. Technical Report, Technical Report PLT-TR-2010-1, PLT Inc., 2010. http://racket-lang. org/tr1.

[10] Sal Khan: Khan Academy. Available at http://www.khanacademy.org/\#computer-science

[11] Xavier Leroy, Damien Doligez, Alain Frisch, Jacques Garrigue, Didier Rémy \& Jérôme Vouillon (2011): The OCaml system release 3.12: Documentation and user's manual. Technical Report, Institut National de Recherche en Informatique et en Automatique.

[12] Miran Lipovaca (2011): Learn You a Haskell for Great Good!: A Beginner's Guide, 1st edition. No Starch Press, San Francisco, CA, USA. Available at http://learnyouahaskell.com

[13] Ben Lippmeier (2010): Gloss. Available at http://gloss .ouroborus.net/.

[14] J Maloney, L Burd, Y Kafai, N Rusk, B Silverman \& M Resnick (2004): Scratch: A Sneak Preview. In: Second International Conference on Creating, Connecting, and Collaborating through Computing, pp. 104109, doi $10.1109 /$ C5.2004.1314376.

[15] Conor McBride \& James McKinna (2004): The view from the left. Journal of Functional Programming 14(1), pp. 69-111, doi $10.1017 /$ S0956796803004829

[16] Ulf Norell (2007): Towards a practical programming language based on dependent type theory. Ph.D. thesis, Chalmers University of Technology.

[17] Simon Peyton Jones, editor (2003): Haskell 98 Language and Libraries: The Revised Report. Cambridge University Press.

[18] Emmanuel Schanzer: Bootstrap. Available at http://www.bootstrapworld.org/

[19] Chris Smith (2011): Haskell for Kids. Available at http://cdsmith.wordpress.com/category/ haskell-for-kids/.

[20] Wouter Swierstra, S. Doaitse Swierstra \& Jurriën Stutterheim (2011): Logisch en Functioneel Programmeren voor Wiskunde D. Technical Report UU-CS-2011-033, Universiteit Utrecht. 